\title{
Ciudad modelo AUDI. ¿Un megaproyecto fallido?
}

\section{AUDI model city. A failed megaproject?}

\section{Resumen}

Adriana Soledad Espinosa-Flores" adrisespinosaf@gmail.com

María de Lourdes Flores-Lucero* maria.flores@correo.buap.mx

Susana Medina-Ciriaco* susanamedinaciriaco@gmail.com

*Benemérita Universidad Autónoma de Puebla

*México
Miudad Modelo es un proyecto que el gobierno del estado de Puebla construyó con la finalidad de fortalecer el desarrollo industrial de la nueva A planta de autopartes AUDI en México. Hoy se habla de que es un proyecto fallido al estar habitado solo en un $15 \%$ de su capacidad total. Pero para conocer si en realidad este proyecto es un quebranto, hemos hecho una evaluación mediante una adaptación de la metodología propuesta por Saltalamacchia y Ziccardi, quienes evalúan el desempeño del gobierno local. Para este caso de estudio la intención de valorar el ejercicio del organismo se basa en los resultados urbanísticos del proyecto Ciudad Modelo, a través de entrevistas, análisis de imágenes satelitales, normativa urbana, y visitas a campo, con la intención de poder apreciar si el desarrollo urbanístico de la ciudad se encuentra en un proceso de consolidación, o en verdad es el anuncio de un proyecto en decadencia.

Palabras clave: megaproyecto; ciudad nueva; política gubernamental; urbanismo sustentable; impacto urbano.

\section{Abstract:}

Model City is a project that the government of the state of Puebla built to strengthen the industrial development of the new AUDI's auto parts plant in Mexico. Today it's said that it's a failed project because it's been habited only $15 \%$ of its total capacity. But to know if this project is a failure, we have made an evaluation that, for this case, was carried out through an adaptation of the methodology proposed by Saltalamacchia and Zicardi, who evaluate the performance of the local government. For this study case, the intention of evaluating the performance of the agency is based on the urban results of the Model City project, based on interviews, analysis of satellite images, urban regulations, and visits to the Model City, with the intention of being able to appreciate if the urban development of the city is in a process of consolidation, or in truth it is the announcement of a project in decline.

Keywords: megaproject; new city; government policy; sustainable urbanism; urban impact. 


\section{Introducción}

Ciudad Modelo es un megaproyecto que surge oficialmente por un ejercicio de planificación regional a partir de una política gubernamental de atracción de nuevas industrias al estado de Puebla. Se buscó la instalación de la armadora alemana AUDI en el municipio de San José Chiapa y para hacer atractiva la propuesta se propuso una ciudad nueva que ofreciera la vivienda, servicios y equipamientos que los trabajadores de la planta pudieran necesitar.

En 2012 el gobierno del estado de Puebla (GEP) impulsó una política de regulación urbana y suelo en San José Chiapa para aprovechar la dinámica de crecimiento e inversión que generaría el proyecto AUDI. Por lo que se aseguró la disposición de la tierra donde hoy se ubica la Planta AUDI y Ciudad Modelo; se modificó la legislación aplicable para asegurar la viabilidad técnica, jurídica y administrativa de estos mismos proyectos; se realizó el Plan Maestro de Ciudad Modelo (GEP, 2015a) que proyecta la construcción de una ciudad nueva sustentable que pretende dar respuesta a la demanda de vivienda, servicios y equipamientos originada por la instalación de la nueva planta automotriz. Para ello se creó un Organismo Público Descentralizado (OPD) para administrar Ciudad Modelo (Decreto de 2017). Dicho proyecto está programado para que se construya en tres etapas, de las cuales hasta el momento solo se tiene edificada la primera, inaugurada a principios de 2017. Sin embargo, a pesar de las grandes expectativas que se anunciaron con este proyecto y de la inversión que se ejerció para construir y tener en funcionamiento a los más de 23 equipamientos y 596 viviendas, la ciudad se encuentra deshabitada, por lo que surge la necesidad y el interés de evaluar el desempeño del OPD que administra este megaproyecto.

\section{Métodos}

La herramienta metodológica que se utilizó para evaluar el desempeño del Organismo Público Descentralizado de Ciudad Modelo es mixta. Se realiza una evaluación de resultados a partir de los objetivos que planteó el organismo. De estos objetivos se definieron las variables a revisar y la manera de operacionalizarlas, es decir, se eligieron las principales dimensiones: eficacia, eficiencia y calidad, que distinguen a los objetivos, y se precisaron los indicadores correspondientes a cada dimensión para obtener la comparación de los resultados obtenidos con los propuestos. La evaluación se realizó con información documental de acuerdo con los objetivos planteados, asi como con los resultados que generó el organismo hasta diciembre de 2020. Por lo que la obtención de información fue a través de la revisión bibliográfica y hemero- gráfica, complementado con visitas a Ciudad Modelo, registro fotográfico y entrevistas a informantes clave como empleados de la Dirección de servicios Públicos e Infraestructura, la Dirección de Planeación y Transparencia del OPD Cd. Modelo ${ }^{1}$, y la Dirección del Instituto Interamericano de Tecnología y Ciencias del Agua².

Esta investigación es un estudio de caso que pretende responder si se cumplieron, o no, los objetivos del proyecto Ciudad Modelo, para lo cual es necesario responder las siguientes preguntas: ¿cómo se ha desempeñado el organismo que lleva el proyecto?, ¿cuál es el estatus del desarrollo urbanístico?, y ¿qué impactos ha generado este megaproyecto en la zona? Para ello, la evaluación de la actuación gubernamental es fundamental.

De acuerdo con Rodríguez-Aguilera y García-Vidal (2012) el perfeccionamiento empresarial es el proceso económico más importante que puede llevarla hacia un nuevo estadio; para ello se requiere de un procedimiento para evaluar el desempeño de las empresas que permita identificar sus limitantes para incorporar un proceso de mejora que contribuya a elevar la eficiencia y eficacia de la empresa estatal, por lo que tomaremos en cuenta en la evaluación los indicadores de eficiencia, eficacia, y calidad.

Es así como, se analizará el desempeño a partir del cumplimiento de los objetivos del proyecto que son:

\section{- Consolidar Ciudad Modelo.}

- Ofrecer servicios públicos eficientes, de vanguardia, y autosustentables.

Para el primer objetivo, se entenderá:

- Eficacia: como el grado de cumplimiento del objetivo, observancia en la entrega de los productos de acuerdo con el plan.

- Eficiencia: medios utilizados para lograr la consolidación de Ciudad Modelo. Se evaluará la manera en cómo se tomaron acciones para lograr consolidar Ciudad Modelo.

- Calidad: la efectividad de las acciones para lograr que se consolidara el proyecto. Muestra el resultado de la eficacia y eficiencia que tuvo el gobierno para lograr el cumplimiento del objetivo.

Para el segundo objetivo se entenderá:

- Eficacia: indica el nivel o grado de los resultados obtenidos en relación con los objetivos predefinidos, donde se plantea el problema de la finalidad y no de la realización. La eficacia se materializa implicando la lógica de resultados (Masou, 2011).

Para este caso se medirá el cumplimiento de los objetivos que se exponen en el Plan Maestro, por lo que se evalua-

${ }^{1}$ Organismo con personalidad jurídica y patrimonio propios, el cual tiene a su cargo la consolidación y administración del Polígono de Ciudad Modelo.

${ }^{2}$ Centro de investigación hidrología, tratamiento de aguas y gestión integral del agua. 
rá la cobertura de los servicios, equipamientos, vivienda y consolidación de la ciudad.

- Eficiencia: adaptación de los productos de acuerdo con las dinámicas del mercado para lograr los objetivos. A aplicarlo al segundo objetivo se evaluarán las características básicas que debe tener cada inmueble, de acuerdo con la norma.

- Calidad: qué tan oportunos y accesibles son los bienes y servicios entregados a los usuarios. Se evaluará accesibilidad del inmueble o servicio de acuerdo con el radio de influencia que indica la norma federal. Como la accesibilidad puede darse con diferentes medios de transporte se consideró como ejemplo el uso del auto y el de la bicicleta.

Una vez desarrollada la metodología de evaluación, se utilizó una escala de valoración formulada por Zúñiga (2009), donde a partir de la evaluación se asignó un puntaje en función de los resultados de la aplicación del instrumental, con rangos más estrechos en las condiciones de excelencia y más amplios en las peores condiciones, ya que de acuerdo con Zúñiga, "bastaría una situación negativa cuya insatisfacción paralizaría el funcionamiento del sistema y lo afectaría severamente" (2009, p. 28); por consiguiente, para calificarse como mal , tendría que ser porque no se cuenta con el inmueble, no hay aceptación del proyecto o la construcción, demanda o radio de influencia es apenas poco mayor del 40\%. En contraste, la calificación de muy bien se otorgó cuando está concluida la construcción o el convenio, los involucrados están satisfechos, o las características de los inmuebles cumplen con las Normas Federales. En cuanto a las evaluaciones intermedias, el rango se distribuyó de acuerdo a los extremos, según Zúñiga.

\section{Resultados}

\subsection{La relevancia de los megaproyectos urba- nos}

La investigación toma como punto de partida el concepto de megaproyecto urbano que manejan Altshuler y Luberoff, (2013) y Díaz-Orueta (2009), el cual tiene un alcance regional y un importante impacto sobre la economía y la morfología socioespacial de la zona donde se desarrolle.

La ejecución de los megaproyectos urbanos no es una acción reciente: basta mencionar la edificación de ciudades nuevas como Brasilia, Chandigarh, o la Ciudad Jardín de Letchworth, creadas en el siglo pasado. Sin embargo, la situación económica mundial ha provocado que este tipo de proyectos sean cada vez más escasos o de menor escala. Ahora estos megaproyectos se han enfocado a reforzar la competitividad de las ciudades. De acuerdo con Salinas (2014), las políticas urbanas promocionan e crecimiento económico, abriendo una etapa de cambio al ser ahora una pieza importante del empresarialismo urbano, donde las autoridades locales incentivan la inversión del capital privado.

Díaz argumenta que la investigación referente a los megaproyectos ocupa un lugar importante debido a la ex- pansión de la gobernanza urbana empresarial, favoreciendo la atracción de inversiones matizadas con ciertos cambios medioambientales para reforzar la legitimidad social, incorporando compromisos en materia de vivienda y empleo (Díaz, 2015).

De esta manera se pueden tener proyectos promovidos por funcionarios públicos que, al contar con voluntad política por parte de la federación, logran un apoyo a sus iniciativas y aprovechan las oportunidades de obtener exenciones, fondos adicionales, o modificaciones de leyes.

Entre los principales retos a los que se enfrentan los gobiernos para la ejecución y seguimiento de megaproyectos está el de asegurar la transparencia en todos los procesos urbano-administrativos, ya que como anota Fernández (2006, p. 24), existe una opaca planeación y gestión urbana, hecho que provoca decisiones arbitrarias y actos de corrupción.

\subsection{El cuestionable megaproyecto urbano de AUDI}

Ciudad Modelo es un megaproyecto que ofrecería los servicios, equipamiento y vivienda que presuntamente se requerirían con la puesta en marcha de la armadora AUDI, lo que no solamente beneficiaría a los empleados de la fábrica, también a los habitantes de la región.

Para asegurar la construcción del megaproyecto urbano, se elaboraron dos instrumentos de planeación: el Programa Subregional de Desarrollo Urbano Sustentable (GEP, 2015) y el Plan Maestro Ciudad Modelo (GEP, 2015a).

El primero, pretendía establecer el ordenamiento territorial sustentable de la subregión conformada por los municipios de San Hipólito Soltepec, Mazapiltepec de Juárez, Nopalucan de la Granja, Rafael Lara Grajales y San José Chiapa, pero al desarrollar el diagnóstico se presenta un ejercicio de demanda de servicios y vivienda que presupone serán solventados con Ciudad Modelo.

Se habla de las oportunidades de empleo con la construcción de la planta, pero no se presentan datos como e tipo de estudios con que cuenta la población para identificar las posibilidades de inserción a la armadora.

También presuponen, sin estudios profundos, que por cuestión de costos por desplazamiento la mayoría de los empleados de AUDI decidirán residir cerca de la planta, ya que la ciudad de Puebla queda a $60 \mathrm{~km}$.

El segundo instrumento de planeación que se elaboró fue el Plan Maestro de Ciudad Modelo, el cual pretende solventar la futura demanda de vivienda que generará la instalación de la nueva industria automotriz, la cual sería planteada como un espacio urbano sustentable, económicamente competitiva y socialmente integral.

Este proyecto se inicia el 30 de septiembre de 2016 y pretendían que contara con los siguientes inmuebles (Tabla 1): 


\begin{tabular}{|c|c|c|}
\hline \multirow{2}{*}{\multicolumn{2}{|c|}{$\begin{array}{l}\text { SISTEMAS } \\
\text { EQUIPAMIENTO GENERAL }\end{array}$}} & \multirow{3}{*}{$\begin{array}{l}\text { SUPERFICIE (m2) } \\
\mathbf{6 1 7 , 4 4 0} \\
28093 \\
\end{array}$} \\
\hline & & \\
\hline EQ-01 & Central de Autobuses* & \\
\hline EQ-02 & Centro de Convenciones* & 16366 \\
\hline EQ-03 & Hospital & 30492 \\
\hline EQ-04 & $\begin{array}{l}\text { Centro de Salud con Servicios Ampliados } \\
\text { CESSA* }\end{array}$ & 3002 \\
\hline EQ-05 & Centro Integral de Servicio CIS* & 1504 \\
\hline EQ-06 & Centro Escolar* & 78144 \\
\hline EQ-07 & Polideportivo Cubierto & 12876 \\
\hline EQ-08 & $\begin{array}{l}\text { Consejo Nacional de Ciencia y Tecnología } \\
\text { (CONACYT)* }\end{array}$ & 11164 \\
\hline EQ-09 & Universidad Bilingüe* & 181168 \\
\hline EQ-10 & Centro Deportivo* & 71919 \\
\hline EQ-11 & $\begin{array}{l}\text { Complejo Regional Centro Sede San José } \\
\text { Chiapa (BUAP)* }\end{array}$ & 182711 \\
\hline \multicolumn{2}{|c|}{ EQUIPAMIENTO BARRIAL } & 22045 \\
\hline EQ-B-01 & Telmex & 175 \\
\hline EQ-B-02 & Estacionamiento & 1772 \\
\hline EQ-B-03 & Mercado 1 & 2445 \\
\hline EQ-B-04 & Jardín Vecinal 1* & 3159 \\
\hline EQ-B-05 & Centro Social Popular & 1989 \\
\hline EQ-B-06 & $\begin{array}{l}\text { Medicina Familiar del Instituto Mexicano } \\
\text { del Seguro Social IMSS* }\end{array}$ & 279 \\
\hline EQ-B-07 & Guardería* & 139 \\
\hline EQ-B-08 & Jardín Vecinal 2 & 1481 \\
\hline EQ-B-09 & $\begin{array}{l}\text { Centro de Salud Urbano de la Secretaría de } \\
\text { Salud SSA }\end{array}$ & 491 \\
\hline EQ-B-10 & Mercado 2 & 2386 \\
\hline EQ-B-11 & Biblioteca & 475 \\
\hline EQ-B-12 & Casa de la Cultura & 1455 \\
\hline EQ-B-13 & Jardín Vecinal 3 & 2783 \\
\hline EQ-B-14 & Comandancia de Policía & 1746 \\
\hline EQ-B-15 & Centro Comunitario Iglesia & 1270 \\
\hline \multicolumn{2}{|c|}{ ZONAS VERDES Y ESPACIO PÚBLICO } & 264104 \\
\hline EL-01 & Espacio Libre 1 & 2146 \\
\hline EL-02 & Espacio Libre 2 & 2029 \\
\hline EL-03 & Espacio Libre 3 & 5913 \\
\hline EL-04 & Espacio Libre 4 & 2100 \\
\hline PL-01 & Parque Lineal $1^{*}$ & 11950 \\
\hline PL-02 & Parque Lineal 2 & 60444 \\
\hline PL-03 & Parque Lineal 3 & 34497 \\
\hline PL-04 & Parque Lineal 4 & 13821 \\
\hline PL-05 & Parque Lineal 5 & 17033 \\
\hline PL-06 & Parque Lineal 6 & 9072 \\
\hline PL-07 & Parque Lineal 7 & 21046 \\
\hline PL-08 & Parque Lineal 8 & 20094 \\
\hline PL-09 & Parque Lineal 9 & 12654 \\
\hline PM-01 & Parque Metropolitano* & 51305 \\
\hline \multicolumn{2}{|c|}{ VIALIDADES } & 315680 \\
\hline VI & Viario* & 305365 \\
\hline RV & Reserva Vial & 10315 \\
\hline
\end{tabular}




\begin{tabular}{|c|c|c|}
\hline SUB-TOTAL & & 1219269 \\
\hline \multicolumn{3}{|c|}{ ACTIVIDADES TERCIARIAS } \\
\hline TE-01 & Hotel $1^{*}$ & 10019 \\
\hline TE-02 & Centro Comercial* & 22619 \\
\hline TE-03 & Hotel 2* & 4958 \\
\hline \multicolumn{2}{|l|}{ SUB-TOTAL } & 37597 \\
\hline \multicolumn{3}{|l|}{ VIVIENDA } \\
\hline HS & Habitacional Subsidiada (2112 viv. ${ }^{*}$ ) & 142306 \\
\hline HT_NS & $\begin{array}{l}\text { Habitacional Tradicional No Subsidiada y } \\
\text { Media ( } 2615 \text { viv. }{ }^{*} \text { ) }\end{array}$ & 239448 \\
\hline $\mathrm{HR}$ & $\begin{array}{l}\text { Habitacional Residencial y residencial Plus } \\
\left(302 \text { viv. }^{*} \text { ) }\right.\end{array}$ & 55692 \\
\hline \multicolumn{2}{|l|}{ SUB-TOTAL } & 437446 \\
\hline \multicolumn{3}{|c|}{ CONEXIONES EXTERNAS } \\
\hline CO-E-01 & Planta potabilizadora* & \\
\hline CO-E-02 & Subestación eléctrica & \\
\hline CO-E-03 & Planta de tratamiento* & \\
\hline CO-E-04 & Punto limpio* & \\
\hline
\end{tabular}

Tabla 1: Total de inmuebles que se programaron construir en Ciudad Modelo

Fuente: Plan Maestro Ciudad Audi (2015)

De acuerdo con el Plan Maestro, en comparación con lo observado en visita de campo, las construcciones que estaban consideradas para la primera etapa que no se han construido son el edificio del $\operatorname{CONACYT}^{3}$ y el $88 \%$ de las viviendas, ya que se pretendía construir 5,029 viviendas y solo se han edificado 596

El proyecto de la ciudad nueva está estructurado por corredores verdes y andadores peatonales que conectan el área habitacional con los equipamientos (Figura 1). De acuerdo con el Plan Maestro, el sistema de corredores estaría diseñado para generar recorridos cortos a pie. Sin embargo, la zona habitacional está aproximadamente de 1400 a 1700 metros de la zona escolar, lo que ya no lo hace confortable para caminar. En el siguiente croquis se muestra la estructura urbana de Ciudad Modelo, su zona habitacional, equipamientos, parques, y vialidades con los que cuenta.

A pesar de que prácticamente se construyeron todos los equipamientos que se tenían programados para la primera etapa, Ciudad Modelo no ha sido un proyecto exitoso para los desarrolladores de vivienda. De acuerdo con el Plan Maestro y el Programa Sub-regional de Desarrollo Urbano Sustentable se proyectaba que con la puesta en marcha de la planta automotriz AUDI, se requeriría de mano de obra cualificada que, en su mayoría (92\%), vendrían de la ciudad de Puebla, por lo que buscarían como lugar de residencia las inmediaciones a las instalaciones de la planta para no tener que hacer un recorrido de traslado diario de más de dos horas.

Además, se consideró la tendencia de la demanda potencial de trabajadores que ganan entre 2 y 7 Salarios Míni-

\footnotetext{
${ }^{3}$ Institución dedicada a promover el avance de la investigación científica en México

${ }^{4}$ Su equivalente en dólar americano a valor en enero de 2021 es de 13.78 y 48.24 respectivamente
}

mos Mensuales (SMM) ${ }^{4}$ que han accedido a la compra de una vivienda de los segmentos popular y tradicional, para prever el comportamiento que tendrían los futuros trabajadores de la planta con derecho a un crédito del Instituto del Fondo Nacional de la Vivienda para los Trabajadores (INFONAVIT) y que no lo hayan aplicado.

De esta manera, se estimó una demanda de 35,036 viviendas en la sub-región, de la cual se estima que solo el $25 \%$ (8,836 viviendas) se asentará en las localidades existentes debido a que no cuentan con la capacidad en infraestructura y servicios para poder albergar toda la demanda, por lo que el 75\% (26,199 viviendas) restante requeriría de un nuevo asentamiento urbano.

De acuerdo con el Plan Maestro en la primera etapa se construirían 5,029 viviendas, de las cuales $42 \%$ serían de vivienda subsidiada, $52 \%$ de vivienda media y tradicional no subsidiada, y el $6 \%$ restante de vivienda residencial y residencial plus.

De esta propuesta hasta la fecha se han construido 596 viviendas, las cuales son del modelo subsidiado, debido a que no se cuenta con una demanda real. Los trabajadores de AUDI prefieren trasladarse desde Puebla que vivir en la zona, por lo que diariamente se observan caravanas de camiones foráneos que traen a los trabajadores a la planta.

El desinterés por habitar en Ciudad Modelo generó una fuerte crítica hacia el GEP, debido a la inversión que se realizó no solo en la construcción de Ciudad Modelo, sino también por lo que implicó el atraer a la armadora de autos. Se publicó en notas periodísticas que se invirtieron 17,656 millones de pesos mexicanos para realizar obras complementarias en la región donde se ubicó la armadora, más 1,300 millones para la construcción de Ciudad Modelo (López, 16 de junio de 2017). 

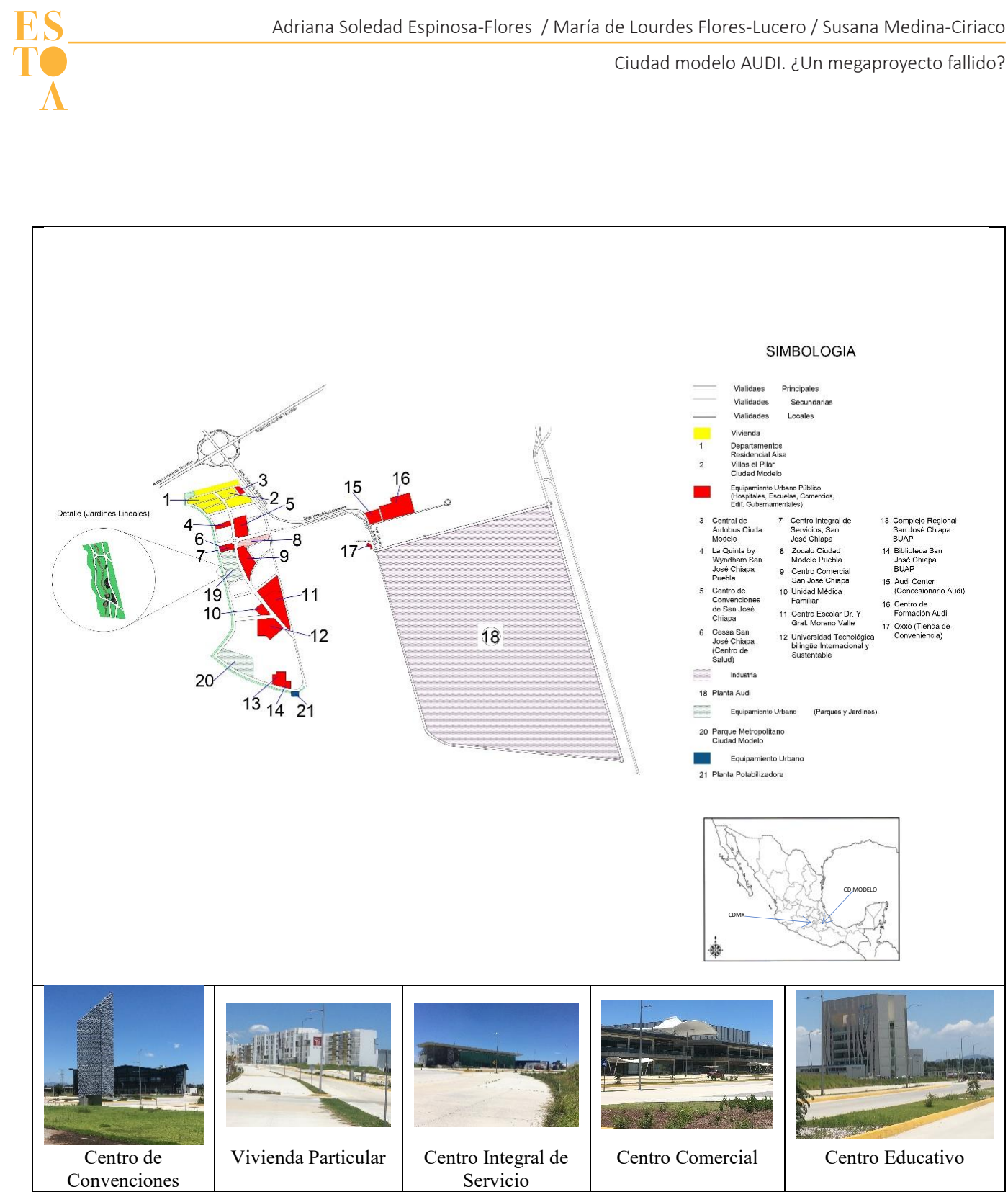

Figura 1. Croquis de la estructura urbana de Ciudad Modelo. Primera etapa Fuente: Elaboración propia con información obtenida en visitas de campo en 2019 y 2020

Ciudad Modelo se proyectó para ser la segunda ciudad más importante del estado de Puebla; sin embargo, la instalación de AUDI por sí sola no es suficiente para que la población sea atraída, por lo que se han tenido que realizar diferentes acciones para consolidar el proyecto, algunas de ellas son:

Para que el Centro Escolar Dr. y Gral. Rafael Moreno Valle contara con un mayor número de alumnos, desde su inauguración el gobierno estatal realizó un convenio con la empresa poblana de transporte Estrella Roja para que algunas de sus unidades sirvan de transporte escolar para trasladar a los alumnos de las localidades de Grajales, Nopalucan y San José Chiapa (López, 16 de junio de 2017). Esta acción contribuyó para que este centro escolar tenga una matrícula de 970 alumnos, distribuidos en los niveles de Primera infancia, Preescolar, Primaria, Secundaria y Bachillerato (Redacción, 14 de febrero de 2019).
En 2018 se realizó convenio con la Secretaría de Marina para que instale la estación naval en Ciudad Modelo, donde se les donó 20 hectáreas para la construcción de desarrollo militar que tendrá una inversión de 160 millones de pesos (Juárez, 26 de agosto de 2018).

Considerando la intención del gobierno federal de desconcentrar la Secretaría de Educación Pública, desde 2018 a la fecha el GEP ha buscado realizar un convenio para que se instalen en Ciudad Modelo, ya que por la cantidad de empleados que atraería la Secretaría de Educación Pública SEP (65 mil trabajadores) sería un gran detonador para el desarrollo del proyecto, pero hasta ahora solo ha quedado en pláticas con el Secretario de Educación Pública (López, 1 de septiembre de 2018; Torres, 13 de diciembre de 2019). 


\subsection{Evaluación de los objetivos expuestos en el Plan Maestro de Ciudad Modelo}

La intención de esta investigación es evaluar los objetivos del Plan Maestro, para ello se tomó como base la metodología de Saltalamacchia y Ziccardi centrada en la evaluación de resultados. Sin embargo, por las condiciones propias del proyecto, al ser una ciudad nueva, con pocos residentes y al observar una desvinculación entre esta ciudad y las localidades aledañas, se hicieron modificaciones a la metodología en función de los indicadores que se podían estudiar, para particularizarlo en el caso de estudio.

El Plan Maestro de Cd. Modelo presenta dos objetivos, los cuales al momento de evaluarlos dan como resultado un desempeño general aceptable, pero entonces ¿por qué Ciudad Modelo en la realidad se vislumbra como un proyecto fallido?

Al evaluar los resultados del proyecto bajo los indicadores de eficacia, eficiencia y calidad, se observa que de manera general se cumplieron.

Al valorar el objetivo de consolidar Ciudad Modelo bajo el indicador de eficacia como grado de cumplimiento en la construcción de los inmuebles se observó que se cumplió parcialmente (Tabla 2), puesto que se construyeron casi todos los equipamientos programados para la etapa 1. En relación con la infraestructura se logró concluir las redes: viales; de agua potable, alcantarillado y saneamiento; energía eléctrica; telefonía e internet, que

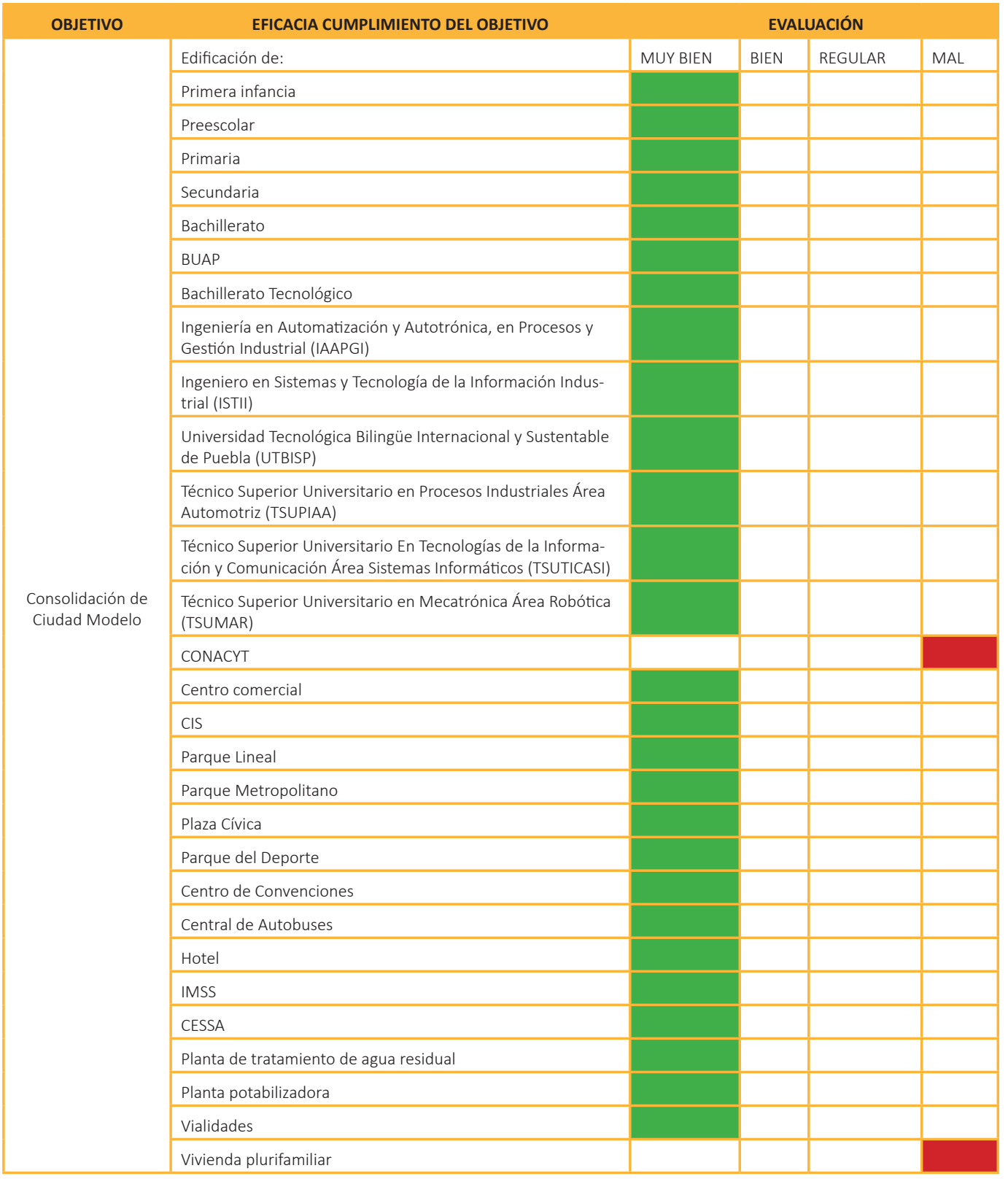

Tabla 2: Evaluación del Objetivo 1 del Indicador Eficacia

Fuente: Elaboración propia con información del Plan Maestro de Ciudad Modelo, visita de campo y revisión de notas periodísticas 
se requieren para el funcionamiento de Ciudad Modelo. Pero con respecto a la vivienda, la proporción de la cantidad de vivienda programada con la edificada es ínfima, a haberse construido solo el $18 \%$.

Al evaluar este mismo objetivo, considerando el indicador de eficiencia como las acciones utilizadas para que se consiguiera la consolidación de Ciudad Modelo, se identificaron varias acciones, de las cuales se concretaron: e Convenio con el grupo AUDI (donde uno de los compromisos para que se instalara la armadora de autos fue la construcción de Ciudad Modelo); la compra del terreno para el proyecto de ciudad; el Convenio con los cinco municipios que están en la región, que se verían impactados de manera más cercana por la construcción de Ciudad Modelo; el Convenio con la Secretaría de Marina para la instalación del laboratorio de Mantenimiento de Optoelectrónica y la construcción de vivienda para los marinos; y la Instalación de la Industria Militar de la Secretaría de la Defensa Nacional. Además de estas acciones están en pláticas un Convenio entre la Cámara Nacional de la Industria de Desarrollo y Promoción de Vivienda (Canadevi), la Secretaría de Economía del GEP y el Director de Ciudad Modelo para la construcción de viviendas con crédito INFONAVIT; y otras que no prosperaron, como el que la Secretaría de Educación Pública se instalara en esta ciudad, siguiendo la política de desconcentración de Gobierno Federal (Tabla 3).

Y, por último, la evaluación con el indicador calidad como la efectividad de las acciones para lograr que se consolidara el proyecto de acuerdo con la opinión de los involucrados en los convenios (Tabla 4). Este punto fue controversial debido a que en un primer momento todos los involucrados en los diferentes convenios expresaron en conferencia de prensa la conformidad de las acciones tomadas con el GEP y el Director de Ciudad Modelo. Sin embargo, en el caso del convenio con los cinco municipios que conforman la región (en enero de 2017), donde se acordó que Ciudad Modelo se hará cargo de los servi- cios de agua potable, drenaje, alcantarillado, tratamiento y disposición de sus aguas residuales; alumbrado público; limpia, recolección, traslado, tratamiento y disposición final de residuos; mercados y centrales de abasto; así como calles, parques y jardines y su equipamiento; además de controlar el otorgamiento de licencias, permisos o autorizaciones para construcción, colocación de anuncios y funcionamiento de comercios y servicios; expedición de licencias de uso de suelo; y la aplicación de los programas regionales, subregionales o programas específicos de ordenamiento ecológico y de desarrollo urbano; los municipios de Soltepec, Mazapiltepec, Nopalucan, Rafael Lara Grajales y San José Chiapa tiempo después se manifestaron en contra de la decisión que habían ejercido y solicitaron al congreso que se les regresaran sus funciones, lo cual lograron parcialmente hasta octubre de 2019.

Con respecto al segundo objetivo de ofrecer servicios públicos eficientes, de vanguardia, y autosustentables, el indicador de eficacia valora el cumplimiento del objetivo en cuanto a la construcción de los equipamientos, infraestructura y vivienda demandados para la primera etapa (Tabla 5). Para conocer la demanda requerida para los inmuebles citados se realizó el Programa Sub-regional de Desarrollo Urbano Sustentable, el cual se apoyó de las Normas de Equipamiento e infraestructura Federales de la Secretaría de Desarrollo Social (SEDESOL), así como un estudio de demanda basado en los empleos que se crearían con la armadora AUDI, los ingresos medios que percibirían los trabajadores y el tamaño medio de los hogares. Se consideró que, de la demanda total de vivienda, un $25 \%$ se repartiría en las localidades existentes, próximas a la planta, pero el resto requeriría de un espacio exprofeso para ellas. Además, se consideró que con base en los perfiles de población de los nuevos residentes y los tipos edificatorios definidos por la Comisión Nacional de Vivienda (CONAVI) se demandaría vivienda subsidiada, media y tradicional no subsidiada, y residencial y residencial plus (GEP, 2015, p. 181).

\begin{tabular}{|c|c|c|c|c|c|}
\hline \multirow{2}{*}{ OBJETIVO } & \multirow{2}{*}{$\begin{array}{l}\text { EFICIENCIA } \\
\text { Medios utilizados: }\end{array}$} & \multicolumn{4}{|c|}{ EVALUACIÓN } \\
\hline & & MUY BIEN & BIEN & REGULAR & MAL \\
\hline \multirow{8}{*}{$\begin{array}{l}\text { Consolidación de } \\
\text { Ciudad Modelo }\end{array}$} & $\begin{array}{l}\text { Convenio con grupo AUDI donde parte de los compromisos } \\
\text { para que se instalara la industria fue el construir Ciudad Mo- } \\
\text { delo }\end{array}$ & & & & \\
\hline & Compra del terreno para el proyecto & & & & \\
\hline & $\begin{array}{l}\text { Convenio con los municipios para la construcción de Ciudad } \\
\text { Modelo }\end{array}$ & & & & \\
\hline & $\begin{array}{l}\text { Construcción de la Etapa } 1 \text { de Ciudad Modelo de acuerdo con } \\
\text { el Plan Maestro }\end{array}$ & & & & \\
\hline & $\begin{array}{l}\text { Pláticas con la Secretaría de Educación Pública para que se ins- } \\
\text { talen en Ciudad Modelo }\end{array}$ & & & & \\
\hline & $\begin{array}{l}\text { Convenio con la Marina para que instalen el laboratorio de } \\
\text { Mantenimiento Optoelectrónica, y vivienda }\end{array}$ & & & & \\
\hline & $\begin{array}{l}\text { Convenio para la Instalación de la Industria Militar de la Secre- } \\
\text { taría de la Defensa Nacional }\end{array}$ & & & & \\
\hline & $\begin{array}{l}\text { Pláticas entre la Canadevi, la Secretaría de Economía de Puebla } \\
\text { y el Director de Ciudad Modelo para la construcción de vivien- } \\
\text { das con crédito INFONAVIT }\end{array}$ & & & & \\
\hline
\end{tabular}

Tabla 3: Evaluación del Objetivo 1 del Indicador Eficiencia

Fuente: Elaboración propia con información del Plan Maestro de Ciudad Modelo, visita de campo y revisión de notas periodísticas 


\begin{tabular}{|c|c|c|c|c|c|}
\hline \multirow{2}{*}{ OBJETIVO } & \multirow{2}{*}{$\begin{array}{l}\text { CALIDAD } \\
\text { Medios utilizados: }\end{array}$} & \multicolumn{4}{|c|}{ EVALUACIÓN } \\
\hline & & MUY BIEN & BIEN & REGULAR & MAL \\
\hline \multirow{4}{*}{$\begin{array}{l}\text { Consolidación de } \\
\text { Ciudad Modelo }\end{array}$} & $\begin{array}{l}\text { Convenio con grupo AUDI donde parte de los compromisos } \\
\text { para que se instalara la industria fue el construir Ciudad Modelo }\end{array}$ & & & & \\
\hline & $\begin{array}{l}\text { Convenio con los municipios para la construcción de Ciudad } \\
\text { Modelo }\end{array}$ & & & & \\
\hline & $\begin{array}{l}\text { Convenio con la Marina para que instalen el laboratorio de } \\
\text { Mantenimiento Optoelectrónica, y vivienda }\end{array}$ & & & & \\
\hline & $\begin{array}{l}\text { Convenio para la Instalación de la Industria Militar de la Secre- } \\
\text { taría de la Defensa Nacional }\end{array}$ & & & & \\
\hline
\end{tabular}

Tabla 4: Evaluación del Objetivo 1 del Indicador Calidad

Fuente: Elaboración propia con información del Plan Maestro de Ciudad Modelo, visita de campo y revisión de notas periodísticas

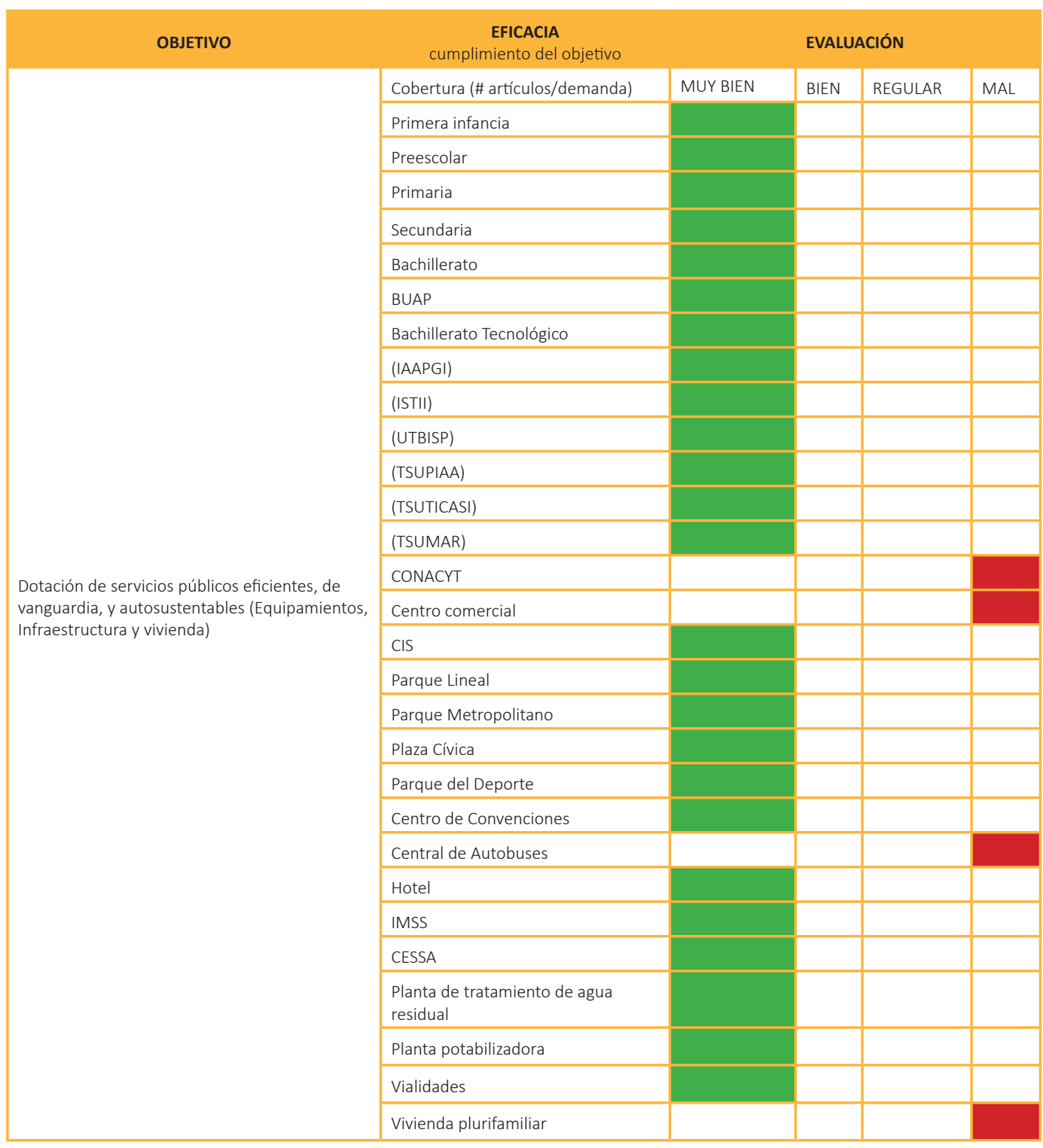

Tabla 5: Evaluación del objetivo 2 del Indicador Eficacia, de los inmuebles programados para la primera etapa Fuente: Elaboración propia con información del Plan Maestro de Ciudad Modelo, visita de campo, revisión de notas periodísticas y entrevistas con informantes clave 
De esta manera, en Ciudad Modelo se construyeron los equipamientos e infraestructura que, de acuerdo a la proyección de población al llegar por la oferta de trabajo y por las normas federales se requerirían para la construcción de la primera etapa del proyecto. En este sentido se concluyó, y está en operación, la infraestructura y casi todos los equipamientos comprometidos, a excepción del edificio del CONACYT y un segundo hotel, que no se construyeron, y en el caso del centro comercial solo está trabajando un local (Notaría Pública) y la central de autobuses se cuenta en desuso.

En relación a la vivienda, se pretendían construir para la primera etapa 5,029 inmuebles, de los cuales se edificaron 596 viviendas plurifamiliares, por lo que se cataloga como un mal cumplimiento del objetivo en relación al indicador de eficacia, si consideramos que de acuerdo a la proyección de demanda se tendrían que construir las más de 5 mil viviendas; pero si hablamos de una deman- da real, la construcción actual de viviendas sobrepasa el interés de compra en Ciudad Modelo, al observarse una importante desocupación de viviendas.

Para evaluar el indicador de eficiencia en relación al segundo objetivo, se revisaron las características arquitectónicas y mobiliario urbano que debe tener cada inmueble, todo ello basado en el Sistema Normativo de Equipamiento Urbano y en el manual de calles de la SEDATU; bajo estos lineamientos el proyecto cumple con los requerimientos de diseño, demanda regional o local, según sea el caso, requerimiento de infraestructura y servicios, distribución de usos de suelo compatible, y construcción de calles completas (Tabla 6). Pero el Plan Maestro también propone que los inmuebles cuenten con elementos sustentables, por lo que expone que para que la vivienda sea sustentable se realizarán acciones que reduzcan la demanda energética mediante el diseño arquitectónico del edificio, incorporando cubiertas vegetales,

\begin{tabular}{|c|c|c|c|c|c|}
\hline OBJETIVO & EFICIENCIA & & EVAL & ACIÓN & \\
\hline \multirow{28}{*}{$\begin{array}{l}\text { Dotación de servicios públicos } \\
\text { eficientes, de vanguardia, y } \\
\text { autosustentables (Equipamien- } \\
\text { tos, Infraestructura y vivienda) } \\
\text { Dotación de servicios públicos } \\
\text { eficientes, de vanguardia, y } \\
\text { autosustentables } \\
\text { (Equipamientos, Infraestructu- } \\
\text { ra y vivienda) }\end{array}$} & $\begin{array}{l}\text { Características arquitectónicas y mobiliario urbano que } \\
\text { debe tener cada inmueble de acuerdo con la norma }\end{array}$ & MUY BIEN & BIEN & REGULAR & MAL \\
\hline & Primera infancia & & & & \\
\hline & Preescolar & & & & \\
\hline & Primaria & & & & \\
\hline & Secundaria & & & & \\
\hline & Bachillerato & & & & \\
\hline & BUAP & & & & \\
\hline & Bachillerato Tecnológico & & & & \\
\hline & (IAAPGI) & & & & \\
\hline & (ISTII) & & & & \\
\hline & (UTBISP) & & & & \\
\hline & (TSUPIAA) & & & & \\
\hline & (TSUTICASI) & & & & \\
\hline & (TSUMAR) & & & & \\
\hline & Centro comercial & & & & \\
\hline & $\mathrm{CIS}$ & & & & \\
\hline & Parque Lineal & & & & \\
\hline & Parque Metropolitano & & & & \\
\hline & Plaza Cívica & & & & \\
\hline & Parque del Deporte & & & & \\
\hline & Centro de Convenciones & & & & \\
\hline & Central de Autobuses & & & & \\
\hline & Hotel & & & & \\
\hline & IMSS & & & & \\
\hline & Planta de tratamiento de agua residual & & & & \\
\hline & Planta potabilizadora & & & & \\
\hline & Vialidades & & & & \\
\hline & Vivienda plurifamiliar & & & & \\
\hline
\end{tabular}

Tabla 6: Evaluación del objetivo 2 del Indicador Eficiencia, de los inmuebles programados para la primera etapa Fuente: Elaboración propia con información del Plan Maestro de Ciudad Modelo, visita de campo y revisión de normas de equipamiento 
espacios vegetales, pasillos de viento, acristalamiento, reflectividad/emisividad, ventilación natural, control solar, inercia térmica, envolvente, orientación, factor de forma, distribución interior de la vivienda, purga nocturna, colchón térmico e iluminación natural. Además de las estrategias pasivas de reducción del consumo de agua potable y estrategias activas de tratamiento y reutilización de agua, con la separación de redes de agua reutilizable (grises y pluviales), filtrado natural, depuración de agua (de manera natural, fotocatalítica, por biorreacción y clorhídrica) y detección de fugas. Pero al revisar estos criterios se apreció, en visita de campo, que no todos se cumplen, como el de la cubierta vegetal en los edificios, para reducir el efecto isla de calor, así como la demanda de refrigeración, por lo que el objetivo de dotación de vivienda autosustentable, considerando los parámetros que la propia Paraestatal propuso en el Plan Maestro de Ciudad Modelo, no se cumplen en su totalidad.

Por último, el indicador de calidad para la evaluación del objetivo 2 permitió observar la accesibilidad de los inmuebles que se ubican en Ciudad Modelo de acuerdo con su radio de influencia (Tabla 7). Los parámetros que se consideraron fueron los de las Normas de Equipamiento Federal, con diferentes modalidades de movilidad según el tipo de equipamiento (estatal, regional, intermedio, medio o básico) y la distancia de la localidad más retirada de la Subregión del Proyecto AUDI, que en este caso es la localidad de Mazapiltepec.

De acuerdo con la Norma, el rango de servicio regional o urbano recomendable se expone en tiempo y distancia, por lo que se hizo el ejercicio con dos tipos de medios de transporte, auto y bicicleta (se excluyó el transporte público al no existir rutas directas de Mazapiltepec a Cd. Modelo). Al realizar la evaluación se observa que la accesibilidad es regular o mala para 12 de los 23 equipamientos, si se quiere llegar a ellos en bicicleta.

Para mejorar la accesibilidad de los equipamientos educativos la Paraestatal ofrece el servicio de transporte escolar, lo que ha permitido la afluencia a estas instituciones y con ello la utilización de dichos equipamientos; sin embargo, el resto de los inmuebles se ven con un acceso limitado porque la mejor opción para llegar a ellos es a través del uso del automóvil, lo que contradice el principio de sustentabilidad que se pretendía con el proyecto.

\begin{tabular}{|c|c|c|c|c|c|}
\hline OBJETIVO & CALIDAD & \multicolumn{4}{|c|}{ EVALUACIÓN } \\
\hline \multirow{24}{*}{$\begin{array}{l}\text { Dotación de servicios públicos } \\
\text { eficientes, de vanguardia, y au- } \\
\text { tosustentables (Equipamientos, } \\
\text { Infraestructura y vivienda) }\end{array}$} & $\begin{array}{l}\text { Accesibilidad del inmueble de } \\
\text { acuerdo con su radio de influencia }\end{array}$ & MUY BIEN & BIEN & REGULAR & MAL \\
\hline & Primera infancia & & en auto & & en bicicleta \\
\hline & Preescolar & en auto & & & en bicicleta \\
\hline & Primaria & & en auto & & en bicicleta \\
\hline & Secundaria & & en auto & & en bicicleta \\
\hline & Bachillerato & en auto & & en bicicleta & \\
\hline & BUAP & en auto y en bicicleta & & & \\
\hline & Bachillerato Tecnológico & en auto & & en bicicleta & \\
\hline & (IAAPGI) & en auto y en bicicleta & & & \\
\hline & (ISTII) & en auto y en bicicleta & & & \\
\hline & (UTBISP) & en auto y en bicicleta & & & \\
\hline & (TSUPIAA) & en auto y en bicicleta & & & \\
\hline & (TSUTICASI) & en auto y en bicicleta & & & \\
\hline & (TSUMAR) & en auto y en bicicleta & & & \\
\hline & Centro comercial & en auto & & en bicicleta & \\
\hline & $\mathrm{CIS}$ & en auto & en bicicleta & & \\
\hline & Parque Lineal & en auto, en bicicleta & & & \\
\hline & Parque Metropolitano & en auto & en bicicleta & & \\
\hline & Plaza Cívica & & en auto & & en bicicleta \\
\hline & Parque del Deporte & & en auto & & en bicicleta \\
\hline & Centro de Convenciones & en auto & en bicicleta & & \\
\hline & Central de Autobuses & en auto & & en bicicleta & \\
\hline & IMSS & & en auto & & en bicicleta \\
\hline & CESSA & & en auto & & en bicicleta \\
\hline
\end{tabular}

Tabla 7: Evaluación del objetivo 2 del Indicador Calidad, de los inmuebles programados para la primera etapa Fuente: Elaboración propia con información del Plan Maestro de Ciudad Modelo y revisión de recorridos

${ }^{5}$ Nota: Los recorridos se realizaron tomando como punto de partida la localidad de Mazapiltepec, que es el municipio, dentro de la región, que queda más retirado de Ciudad Modelo. Distancia Cd. Modelo- Mazapiltepec: $23 \mathrm{Km}$.
Tiempo en automóvil Cd. Modelo- Mazapiltepec: 39 minutos. Tiempo en bicicleta Cd. Modelo- Mazapiltepec: 1 hora con 10 minu tos.

Tiempo caminando para radios de servicios locales: 10 minutos y distancias menores a $750 \mathrm{mt}$ 


\section{Discusión}

De acuerdo con el sitio web Urban Hub, los megaproyectos exitosos deben contar con una buena planificación que incluya un sistema de control seguro y un excelente análisis de riesgo. De acuerdo con ellos, la clave está en incorporar todos los factores involucrados (económico, social e institucional) en las primeras etapas, lo que ayuda a garantizar el retorno de la inversión a largo plazo y beneficios sostenibles y positivos para la sociedad en una ciudad nueva.

Pero ¿qué es lo que está pasando en Ciudad Modelo?, e GEP apostó por un proyecto industrial (armadora AUDI) que llevó a la construcción de Cd. Modelo, debido a que la región no ofrecía las condiciones que requería el futuro personal de AUDI. Se construye un megaproyecto basado en información de una demanda potencial sin considerar la preferencia de las familias de los trabajadores de AUDI que en su mayoría siguen viviendo en la ciudad de Puebla. En este caso el radio de influencia que ejerce Puebla en comparación con $\mathrm{Cd}$. Modelo ha hecho que sean los trabajadores los que estén dispuestos a viajar diariamente a la armadora y regresar a Puebla, tras un recorrido de más de una hora.

Además, se presume de ser una ciudad sustentable, pero se sigue un modelo territorial caracterizado, como dirían del Castillo y Sánchez (2017), por una expansión irracional, sin criterios de optimización, fragmentación social, agotamiento del territorio e incremento de los costos energéticos

A pesar de que $\mathrm{Cd}$. Modelo cuenta con una mejor infraestructura y equipamientos que las localidades aledañas, las viviendas, en su mayoría, siguen sin ocuparse, y por consiguiente los equipamientos están subutilizados. Esto muestra que la intención de que $\mathrm{Cd}$. Modelo generara un impacto positivo para la Subregión no se ha reflejado todavía; a no ser de la oferta educativa, el resto de los equipamientos no han logrado beneficiar a los poblados vecinos.

El trabajo que tiene el gobierno para consolidar este proyecto es arduo y tendrá que seguirse apuntalando con la instalación de más proyectos, no solo bajo la visión de federalismo ascendente, ya que la construcción de inmuebles de la Marina y la Secretaría de la Defensa Nacional, así como la construcción de la zona industrial FINSA ${ }^{6}$ Puebla II, no son suficientes. Se sigue hablando de la posibilidad de que cuando se desconcentre la Secretaría de Educación Pública se instale en Cd. Modelo, pero si no se realizan acciones que hagan más atractiva esta ciudad a comparación de Puebla, los beneficiados van a seguir siendo las líneas de autobuses foráneos y las inmobiliarias en Puebla.

Dicho lo anterior, el desempeño del Organismo Público Descentralizado de Cd. Modelo con relación a los objetivos trazados en su Plan Maestro evaluados bajo resultados urbanísticos obtiene un resultado aceptable, excepto en lograr la consolidación de la ciudad, la cual no está generando beneficios ni a los pobladores vecinos, ni a las

${ }^{6}$ Empresa del sector inmobiliario industrial con mayor cobertura en México. arcas municipales, por lo que hasta el momento podemos clasificarlo como un proyecto fallido.

Si en su momento se pretendió una política urbana bajo una visión empresarial, donde la autoridad incentivara la inversión del capital privado, esto no se ha logrado. En las últimas décadas se ha buscado la asociación público-privado para reducir la carga financiera del Estado, pero para lograrlo hay que contar no solo con mecanismos de control y rendición de cuentas, sino también incluir dentro de los procesos el consenso social y político con excelentes negociadores para que no sea únicamente la iniciativa privada la que obtenga beneficios, y haya un impacto favorable local y regional.

\section{Recomendaciones}

La evaluación al desempeño del gobierno estatal en relación al proyecto Ciudad Modelo, basado en la metodología propuesta por Saltalamacchia y Zicardi, permitieron analizar los resultados urbanísticos del megaproyecto; se observó el interés de los diferentes gobiernos por apoyar la consolidación de la ciudad nueva, reflejándose en la construcción de los equipamientos necesarios para su desarrollo urbano, pero a pesar de ello los trabajadores de la armadora de autos AUDI prefieren vivir en otras localidades a pesar de los gastos de transporte y de tiempo que ello les provoca, por lo que resulta interesante poder complementar la metodología utilizada con otra que valore la parte social, la preferencia de ese mercado que se suponía cautivo por trabajar en la armadora pero que ha demostrado ser autónomo en sus decisiones de estancia, por lo que queda latente la posibilidad de proseguir con la investigación, pero ahora considerando a la población afectada por esta política gubernamental.

\section{Agradecimientos}

Para la realización de este trabajo se contó con el apoyo del Consejo Nacional de Ciencia y Tecnología (CONACYT) del gobierno mexicano, quien auspició la estancia pos-doctoral en la Facultad de Arquitectura de la Benemérita Universidad Autónoma de Puebla.

Cómo citar este artículo/How to cite this article: Espinosa-Flores, A., Flores-Lucero, M. y Medina-Ciriaco, S. (2022). Ciudad modelo AUDI. ¿Un megaproyecto fallido?. Estoa. Revista de la Facultad de Arquitectura y Urbanismo de la Universidad de Cuenca, 11(21), 45-57. https://doi.org/10.18537/ est.v011.n021.a04 


\section{Referencias bibliográficas}

Altshuler, A. y Luberoff. D. (2013). La política cambiante de los megaproyectos urbanos. En M. Smolka y L. Mullahy (Coords), Políticas de suelo urbano (pp. 22-30). Lincoln Institute of Land Policy. https://www.lincolninst. edu/sites/default/files/pubfiles/politicas-de-suelo-urbano-full.pdf

del Castillo, A. y Sánchez Carrera, V. (2017). Propuesta para un modelo europeo de ciudad sostenible. Caso de estudio: plan urbano de la ciudad de Kalmar. Estoa. Revista de la Facultad de Arquitectura y Urbanismo de la Universidad de Cuenca, 6(11), 137-142. https://doi. org/10.18537/est.v006.n011.a10

Díaz Orueta, F. (2009). El impacto de los megaproyectos en las ciudades españolas. Hacia una agenda de investigación. Estudios Demográficos y Urbanos, 24(1), 193 218. https://doi.org/10.24201/edu.v24i1.1348

Díaz Orueta, F. (2015). Megaproyectos urbanos y modelo de ciudad. El ejemplo de Madrid Río. Cuaderno Urbano. Espacio, cultura, sociedad, 19(19), 179-200. http://dx. doi.org/10.30972/crn.1919813

Fernández G., J.M. (2006). Planificación estratégica de las ciudades. Nuevos instrumentos y procesos. Reverté.

GEP. (2015). Programa Sub-regional de Desarrollo Urbano Sustentable. GEP-IDOM. https://pueblacontralacorrupcion.org/wp-content/uploads/2019/10/31-PROGRAMA-SUBREGIONAL-IDOM-Nov-15.pdf

GEP. (2015a). Plan Maestro Ciudad Modelo. Primera etapa. GEP-IDOM

Decreto de 2017 [Honorable Congreso del Estado de Puebla]. Que crea el Organismo Público Descentralizado denominado Ciudad Modelo. 27 de enero de 2017. Orden Jurídico Poblano. https://pueblacontralacorrupcion org/wp-content/uploads/2019/10/7-DECRETO-CONGRESO-CREA-EL-OPD_CIUDAD_MODELO.pdf

Juárez, V. (26 de agosto de 2018). Incorporan Estación Naval en Ciudad Modelo Audi. El Sol de Puebla. https:// www.elsoldepuebla.com.mx/local/estado/incorporan-estacion-naval-a-ciudad-modelo-audi-1945292.html

López, E. (16 de junio de 2017). Como pueblo fantasma, así luce la ciudad Modelo Audi de Moreno Valle. Central. https://www.periodicocentral.mx/2017/gobierno/item/9788-como-pueblo-fantasma-asi-luce-la-ciudad-modelo-audi-de-moreno-valle-fotos-y-video\#ixzz5 mFymgU7K

López, E. (1 de septiembre de 2018). Tony Gali propone Ciudad Modelo Audi para que vivan trabajadores de la SEP. Central. https://www.periodicocentral.mx/2018/gobierno/item/19969-tony-gali-propone-ciudad-modeloaudi-para-que-ahi-vivan-trabajadores-de-la-sep

Masou, R. (2011). La reforma presupuestaria francesa: ¿Cómo mantener un discurso sobre la calidad mientras la mayoría de los indicadores se centran en la eficacia?
Revista de Estudios de la Administración Local y Autonómica, (315-316), 215-234. https://doi.org/10.24965/reala.vi315-316.100428

Rodríguez-Aguilera, A., y García-Vidal, G. (2012). Eficacia y eficiencia, premisas indispensables para la competitividad. Ciencias Holguin, 18(3), 1-14.

Salinas-Arreortua, L. (2014) Empresarialismo y transformación urbana. El caso de la ciudad de México. ANDULI. Andanza de Ciencias Sociales, 13(1), 59-74. http://dx.doi. org/10.12795/anduli.2014.i13.04

Saltalamacchia, H. y Ziccardi, A. (2005). Las ciudades mexicanas y el buen gobierno local: una metodología para su evaluación. Revista Mexicana de Sociología, 67(1), 31-97. http://dx.doi.org/10.22201/ iis.01882503p.2005.001.6016

SEDATU-BID. (2019). Manual de Calles. Diseño vial para calles mexicanas. https://www.gob.mx/cms/uploads/attachment/file/509173/Manual_de_calles_2019.pdf

SEDESOL. (5 de septiembre de 2018). Sistema Normativo de Equipamiento Urbano. Manual. http://normateca.sedesol.gob.mx/es/SEDESOL/Documentos

Torres, I. (13 de diciembre de 2019). Ciudad Modelo o Huejotzingo posibles sedes para la SEP federal. Central. https://contrastesdepuebla.mx/ciudad-modelo-o-huejotzingo-posibles-sedes-para-la-sep-federal

Redacción. (14 de febrero de 2019). Centro Escolar Doctor y General Rafael Moreno Valle es de excelencia. UN1ÓN Puebla. https://www.unionpuebla.mx/articulo/2019/02/14/educacion/centro-escolar-doctor-y-general-rafael-moreno-valle-es-de-excelencia

Urban Hub. (9 de marzo de 2020). Cities. https://www. urban-hub.com/es/

Zúñiga, A. (2009). Indicadores para la evaluación de la calidad ambiental del hábitat urbano. Revista científica Nexo. 22(1), 23-31. https://doi.org/10.5377/nexo. v22i1.41 